\title{
Deformation and fracture of adhesive layers constrained by plastically-deforming adherends
}

\author{
M. S. KAFKALIDIS ${ }^{1}$, M. D. THOULESS ${ }^{1,2, *}$, Q. D. YANG ${ }^{1}$ and S. M. WARD ${ }^{3}$ \\ ${ }^{1}$ Department of Mechanical Engineering and Applied Mechanics, University of Michigan, \\ Ann Arbor, MI 49109, USA \\ ${ }^{2}$ Department of Materials Science and Engineering, University of Michigan, Ann Arbor, \\ MI 49109, USA \\ ${ }^{3}$ Ford Research Laboratory, Ford Motor Company, Dearborn, MI 48121, USA
}

Received in final form 3 March 2000

\begin{abstract}
The use of an embedded-process zone (EPZ) model to investigate the mode I cohesive parameters for plastically-deforming, adhesively-bonded joints is demonstrated in this paper. It is shown that for the particular systems investigated, the cohesive parameters are consistent with an adhesive layer deforming in accordance with its bulk constitutive properties (as constrained by the adherends). In other words, these systems provide examples where the cohesive tractions exerted by an adhesive layer can be calculated simply from considerations of the constrained deformation of the adhesive. Consistent with such calculations, the peak stress in the adhesive layer decreases as the level of the constraint decreases (either with an increase in the thickness of the adhesive layer or with a decrease in the thickness of the adherends). It is also shown that owing to a compensating effect in which the critical displacement for failure varies with the constraint, the energy absorbed by the adhesive layer (the 'intrinsic' toughness of the joint) is essentially independent of the geometry in these systems.
\end{abstract}

Keywords: Fracture; plasticity; adhesive layers; adhesive joints; process zone.

\section{INTRODUCTION}

The fracture of an interface between two materials depends on the geometry, the constitutive properties of the adherends, and the details of the bonding across the interface. The relationship between the normal cohesive stresses and the normal displacements across the interface dictates the failure of a joint loaded in an opening mode $[1,2]$. Two characteristics of the relationship are of particular importance: the area under the stress-displacement curve, which is designated the 'intrinsic' toughness of the interface, $\Gamma_{\mathrm{o}}$, and the peak opening stress, $\hat{\sigma}$, which represents

\footnotetext{
*To whom correspondence should be addressed. E-mail: thouless@engin.umich.edu
} 
the cohesive strength of the interface. If the deformation of the bonded system is dominated by linear elasticity, $\Gamma_{\mathrm{o}}$ is the only parameter required to characterize fracture. Conversely, if the conditions of linear-elastic fracture mechanics do not apply, then the peak cohesive stress, $\hat{\sigma}$, provides an additional parameter that plays a crucial role in determining the failure of the joint [3]. In particular, the parameter $\Gamma_{\mathrm{o}}$ alone cannot be used to characterize fracture when the adherends deform in a plastic fashion; both $\Gamma_{\mathrm{o}}$ and $\hat{\sigma}$ control the fracture process. (The precise details of the shape of the cohesive law have a minor influence on the fracture behavior, and this effect can generally be ignored [3].)

It is possible to view the role of an adhesive layer solely as one of providing the required cohesive tractions between two adherends. The 'intrinsic' toughness of the joint, $\Gamma_{\mathrm{o}}$, is then associated with the energy dissipated within the adhesive layer (per unit area of crack advance). It is distinct from the total energy absorbed by the joint per unit area of crack advance, which includes the contribution of the plastic deformation of the adherends. The precise shape of the traction-separation law across the adhesive layer, and the magnitudes of $\Gamma_{\mathrm{o}}$ and $\hat{\sigma}$, depends on the constitutive properties and thickness of the adhesive layer, the loading rate, and any constraint effects induced by the geometry. Yang et al. [4, 5] have demonstrated that the use of these two parameters is an excellent way of quantifying the properties of adhesive layers. In particular, by using this approach, detailed quantitative predictions about the strength and deformation of plastically-deforming joints were made for the first time. Experimental results from various mode I geometries with different thicknesses of adherends were compared with numerical predictions using a single value for each of the two parameters, and excellent agreement was found in all cases $[4,5]$.

The approach discussed above involves the adaptation of a numerical technique known as an embedded-process zone (EPZ) model [3,6]. The complete elasticplastic properties of the adherends are modeled by a standard finite-element code. The adhesive layer is modeled by elements that serve to represent a cohesive traction-separation law similar to that of Fig. 1. In this approach, the role of the adhesive is reduced to one of merely providing tractions across the interface. The form of the traction-separation law provided by the adhesive layer and, in particular, the values of $\Gamma_{\mathrm{o}}$ and $\hat{\sigma}$ are assumed to be characteristics of a particular adhesive layer in its constrained configuration at a given loading rate. The numerical calculations proceed by the application of the appropriate loads, and the fracture and deformation evolve naturally depending on the chosen values of the two fracture parameters, $\Gamma_{\mathrm{o}}$ and $\hat{\sigma}$. Yang et al. [4] deduced values for these parameters using an iterative process in which repeated calculations were performed for one particular geometry and compared with the experimental observations for that geometry until a good match was obtained. It was the self-consistency between subsequent predictions based on these values and associated experimental results that validated the significance of the choice of parameters. 


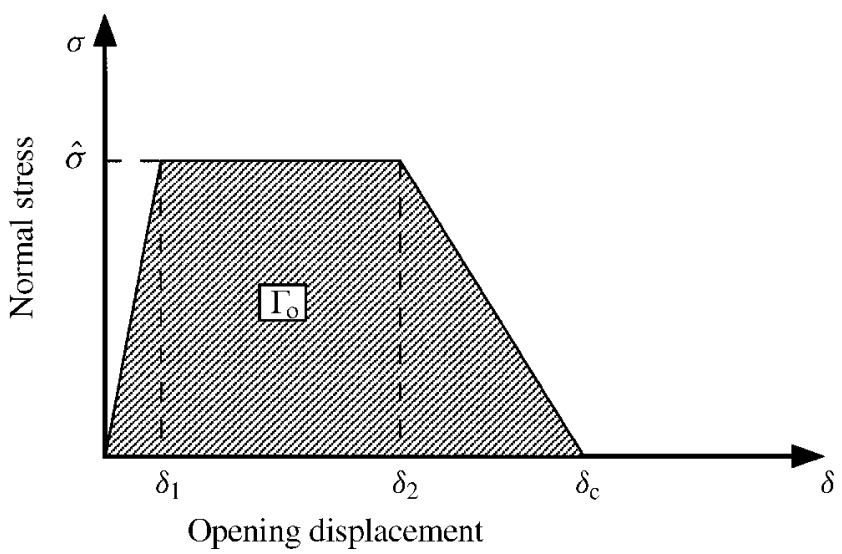

Figure 1. Schematic plot of the normal stress vs. opening displacement for the cohesive tractions across an interface. The area under the curve is the 'intrinsic' toughness of the interface and is denoted by $\Gamma_{\mathrm{o}}$. The cohesive strength of the interface is denoted by $\hat{\sigma}$.

The work described in the present paper was undertaken to explore whether the fracture parameters could be related to the physical properties of the adhesive. It was of particular interest to understand how these parameters might depend on the constitutive properties of the adhesive, the constraint exerted by the adherends on the adhesive layer, and the thickness of the adhesive layer. To this end, this work was divided into three portions. In the first portion, continuum finite-element calculations were conducted to examine how the opening stresses and displacements associated with the deformation of an adhesive layer depended on the magnitude of the crack-driving force (or energy-release rate) and the geometry. In the second portion, experiments were performed in which double-cantilever beam specimens were fractured by means of a wedge inserted down the interface. In the third portion, these experiments were analyzed using an EPZ model to determine the fracture parameters. The effects of varying either the thickness of the adherends or the thickness of the adhesive layer were studied for two commercial adhesives.

\section{RESULTS}

As discussed above, the starting premise of this work is that the role of the adhesive is to provide a traction-separation relationship across the interface and that this relationship is dictated by the deformation of the adhesive layer subject to the constraint exerted on it by the adherends. Therefore, the first step in this study involved conducting continuum finite-element calculations to determine the behavior of adhesive layers constrained between two plastically-deforming adherends.

\subsection{Continuum finite-element calculations}

The basic geometry used for the calculations and experiments is shown in Fig. 2. The joints were symmetrical, consisting of two metal sheets (either aluminum or 


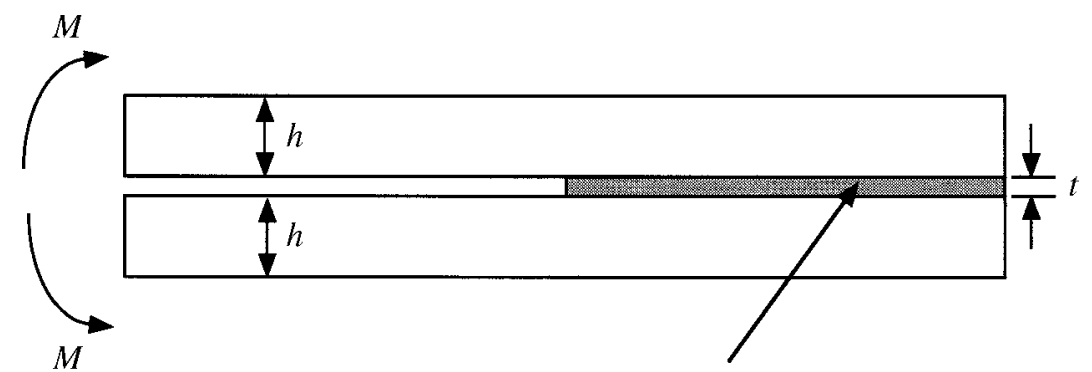

Adhesive layer

Figure 2. The basic geometry used for the calculations and experiments. The joints are symmetrical and consist of two thin metal sheets bonded by a uniform layer of a commercial adhesive. The joints are opened by an applied moment.

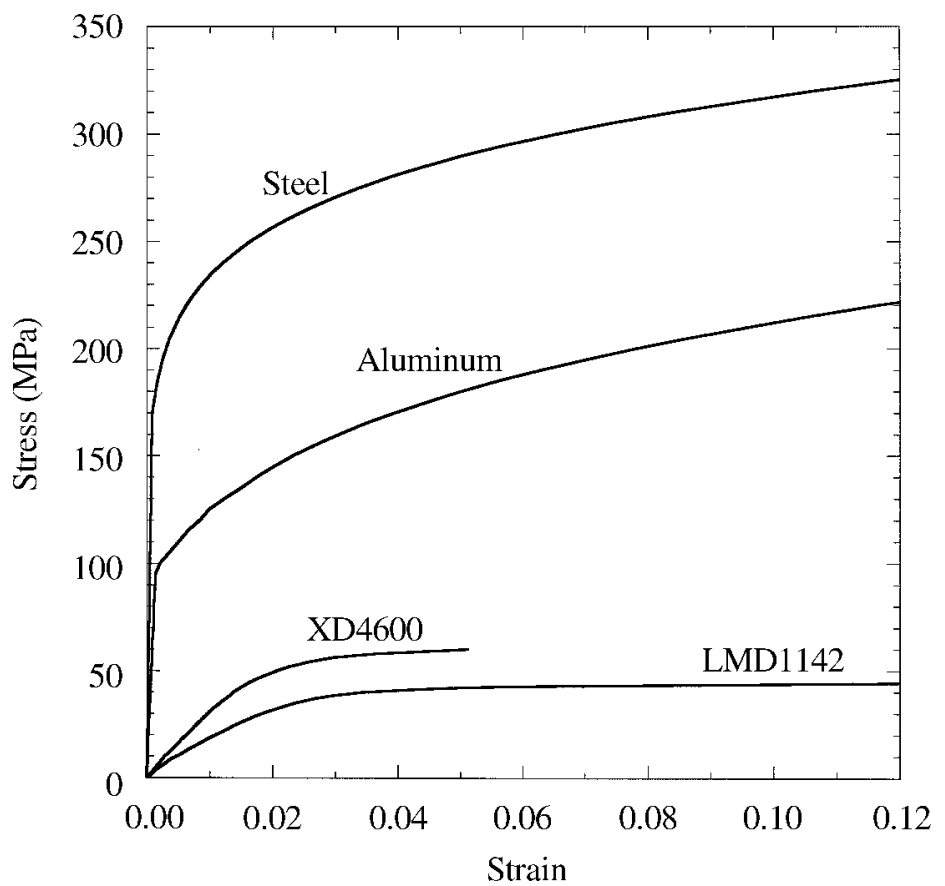

Figure 3. Bulk stress-strain curves for the two commercial adhesives and the adherends used in this study. The curves for the adhesives correspond to a strain rate of approximately $10^{-2} \mathrm{~s}^{-1}$. The data for the Ciba Specialty Products XD4600 are replotted by courtesy of Ciba Specialty Products Inc. The data for Ciba Specialty Products LMD1142 are derived from the results of Duncan et al. [8].

steel) bonded by a uniform layer of a commercial adhesive, and they were loaded by a moment applied to each arm by means of a wedge inserted down the interface. Two initial assumptions were made about the deformation of the adhesive layer: (i) the constraint exerted by the adherends did not cause a fundamental change in the deformation mechanisms of the adhesive layer, and (ii) the bulk properties of the adhesive could be used, with a reasonable degree of accuracy, to describe the 
properties of a layer between $100 \mu \mathrm{m}$ and $1 \mathrm{~mm}$ thick. Subsequent comparisons between the predictions based on these assumptions and the predictions of the EPZ models allowed the validity of the assumptions to be assessed. Representations of the bulk uniaxial stress - strain curves for the two adhesives studied in this paper are shown in Fig. 3. ${ }^{1}$ These curves correspond to a strain rate of about $10^{-2} \mathrm{~s}^{-1}$, which has been determined to be appropriate for the experimental studies described later. For the purposes of the numerical modeling, the curves were approximated by a point-wise representation. ${ }^{2}$ In addition, Poisson's ratio was taken to be 0.35 for both adhesives and it was assumed that the multi-axial yield was governed by a pressure-independent von Mises criterion. ${ }^{3}$ The adherends were modeled as elasticplastic materials with isotropic power-law hardening after yield, and representative constitutive properties of these materials, obtained from uni-axial tensile tests, were used in the finite-element calculations.

Since the traction-separation relationship across the interface was of particular interest, the normal (opening) stress distribution in the adhesive layer and the displacements across the layer were determined as a function of $\mathcal{G}$ (defined here as the energy release that acts on the adhesive layer, so that fracture occurs when $\mathcal{G}=\Gamma_{\mathrm{o}}$ ). Examples of the normal stress distribution and the corresponding displacements are shown in Figs $4 \mathrm{a}$ and $4 \mathrm{~b}$ for several different values of $\mathcal{G}$. These results show that tensile stresses are always limited to a region that extends about $1 \mathrm{~mm}$ ahead of the crack and that the stresses peak at a distance of about one bondline thickness ahead of the crack before dropping fairly rapidly. The magnitude of the peak stress, $\hat{\sigma}$, rises with $\mathcal{G}$. The precise details of the relationship between the two quantities depend on the constraint exerted by the adherends on the adhesive, as represented by the ratio of the bond-line thickness to the adherend thickness. Examples of how $\hat{\sigma}$ varies with $\mathcal{G}$ for different levels of constraint are shown in Fig. 5.

\subsection{Experimental results}

Specimens were fabricated using the two commercial adhesives characterized in Fig. 3. Three separate sets of specimens were prepared: (i) aluminum-aluminum bonds with the XD4600 adhesive; (ii) steel-steel bonds with the XD4600 adhesive; and (iii) aluminum-aluminum bonds with the LMD1142 adhesive. Details of the specimen preparation have been described elsewhere [7] and identical procedures were followed in this study. The thickness of the adhesive layer, which was measured optically after fabrication, was kept constant in each specimen by using

\footnotetext{
${ }^{1}$ XD4600 and LMD1142 were from Ciba Specialty Products.

${ }^{2}$ Extrapolation of the data was used when it was determined that the failure strain in the constrained layer was larger than that obtained in the bulk.

${ }^{3}$ The important deformation near the crack tip occurs under conditions of hydrostatic tension. Therefore, any difference between the compressive yield stress and the tensile yield stress is not expected to have a major influence on the crack-tip deformation.
} 


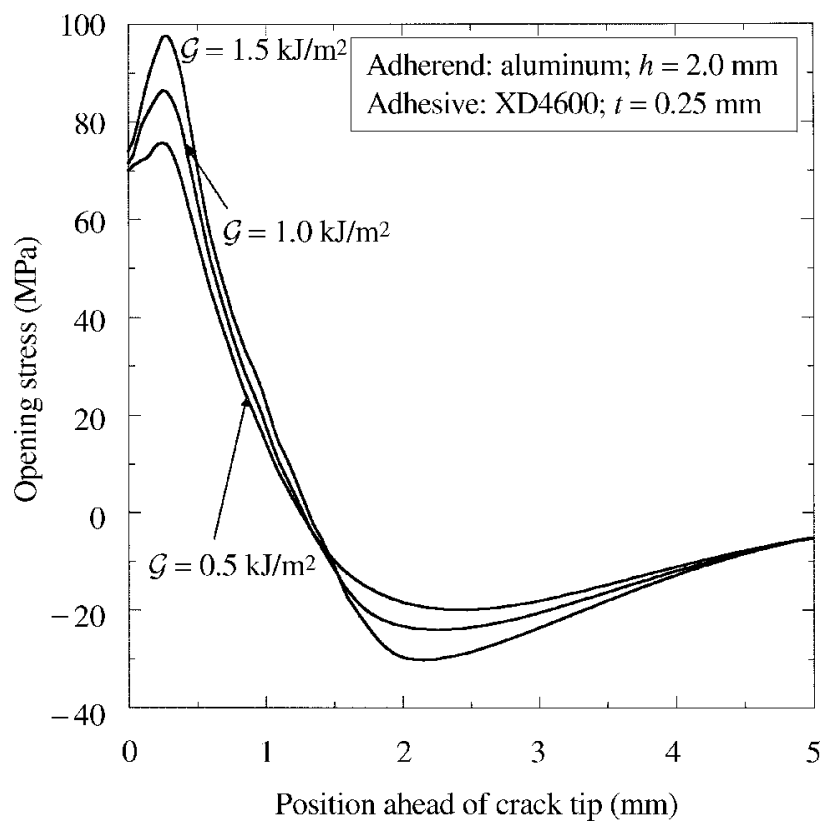

(a)

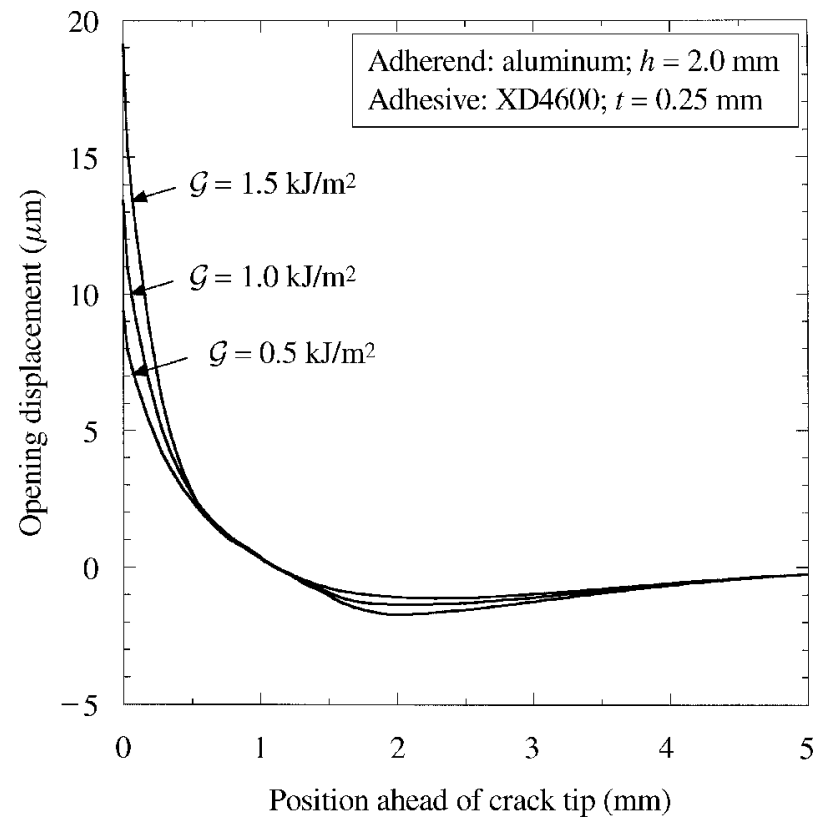

(b)

Figure 4. (a) Distribution of the opening stresses (exerted on the adherends by the adhesive layer) ahead of the crack, plotted at different values of $\mathcal{G}$ (the local energy-release rate acting on the adhesive layer). These calculations were done for $2.0 \mathrm{~mm}$ thick aluminum adherends and a $0.25 \mathrm{~mm}$ thick XD4600 adhesive layer. (b) The opening displacements (across the adhesive layer) corresponding to the data of a. 


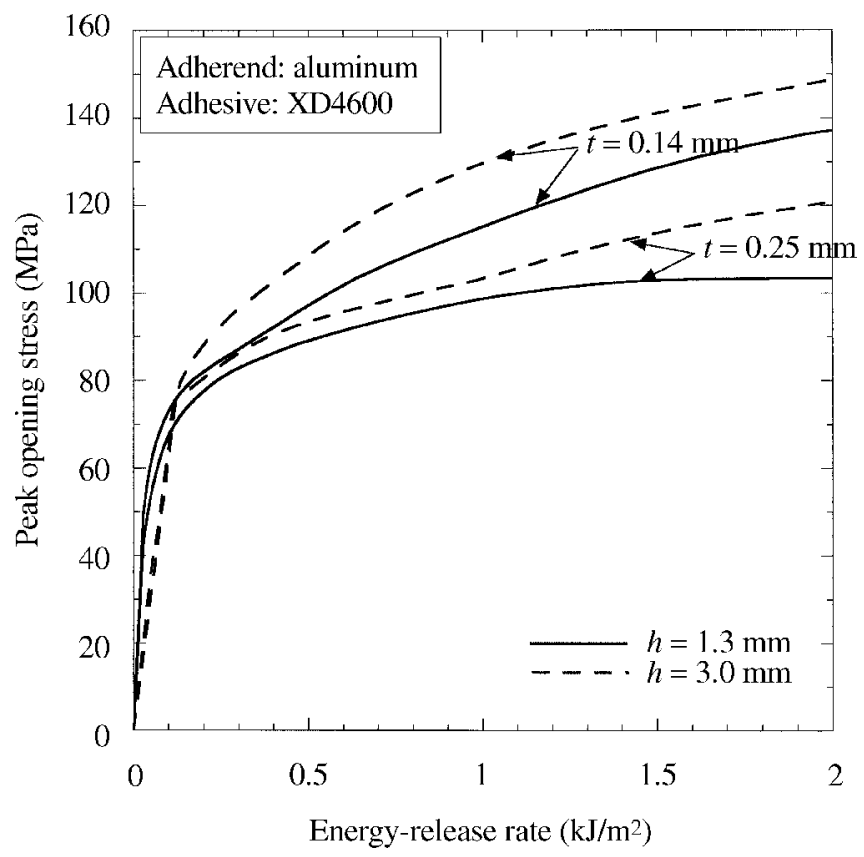

(a)

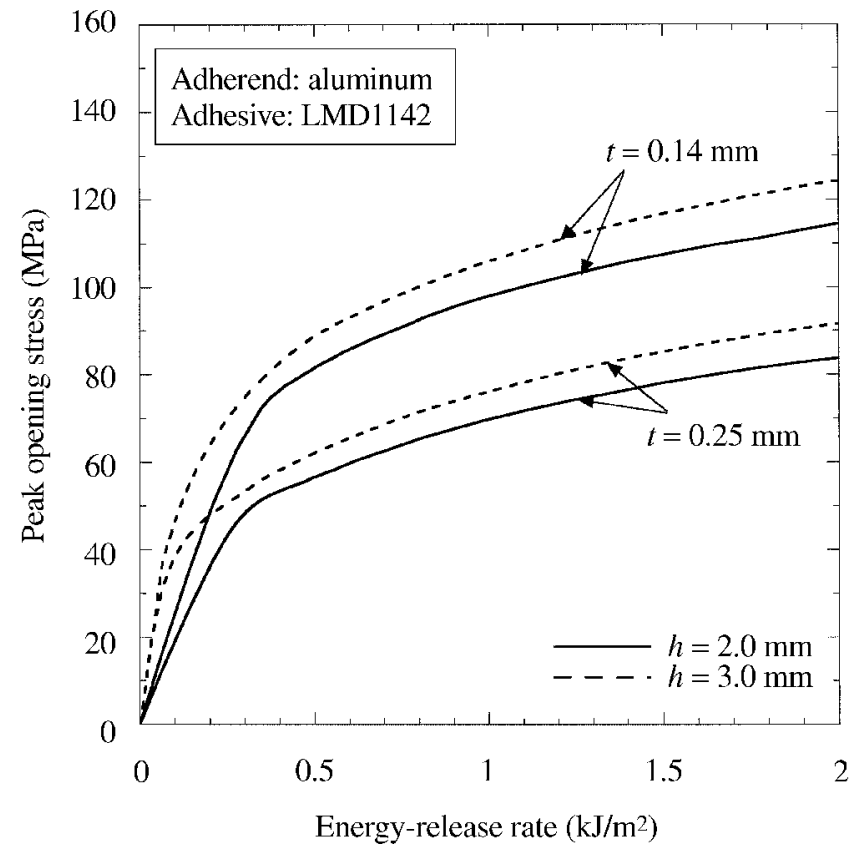

(b)

Figure 5. Predicted relationship between the peak opening stress and energy-release rate for a layer of (a) XD4600 and (b) LMD1142 bonded to aluminum. The thickness of the adhesive layer is denoted by $t$ and the thickness of the adherends by $h$. 


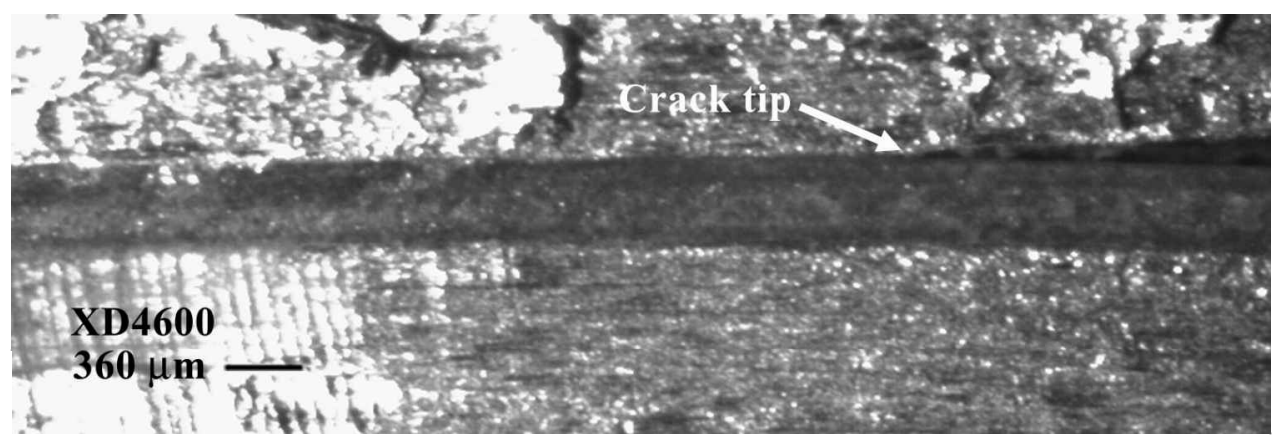

(a)

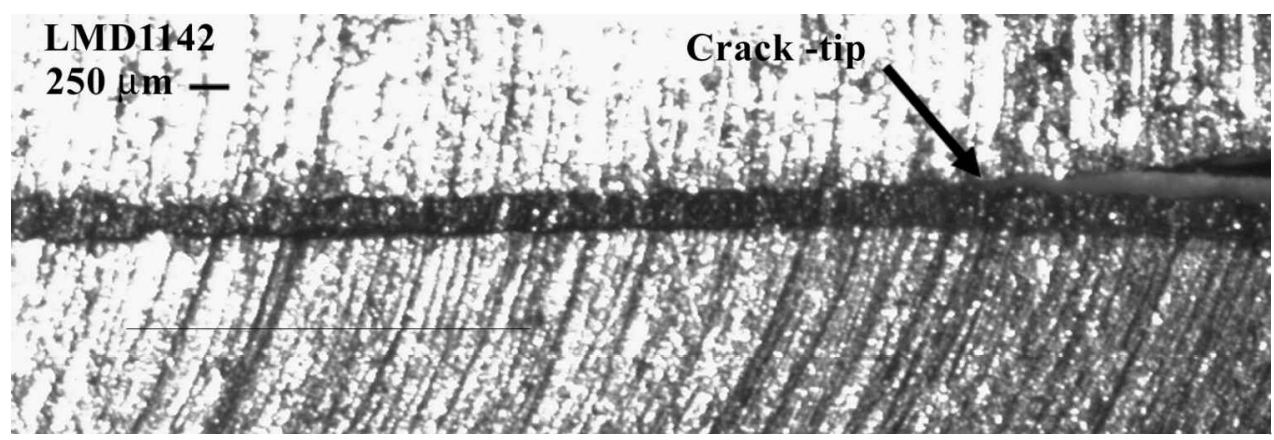

(b)

Figure 6. Optical micrographs of the crack-tip region in (a) a $360 \mu \mathrm{m}$ thick layer of XD4600 bonded to $3.0 \mathrm{~mm}$ thick aluminum and (b) a $250 \mu \mathrm{m}$ thick layer of LMD1142 bonded to $2.0 \mathrm{~mm}$ thick aluminum.

\section{Table 1.}

Process parameters for the XD4600 adhesive

\begin{tabular}{lllllr}
\hline $\begin{array}{l}\text { Adhesive } \\
\text { layer } \\
\text { thickness }\end{array}$ & $\begin{array}{l}\text { Adherend } \\
\text { thickness } \\
t(\mathrm{~mm})\end{array}$ & Adherend & $\begin{array}{l}\text { Radius of } \\
\text { curvature } \\
(\mathrm{mm})\end{array}$ & $\begin{array}{l}\text { 'Intrinsic' } \\
\text { toughness } \\
\Gamma_{\mathrm{o}}\left(\mathrm{kJ} / \mathrm{m}^{2}\right)\end{array}$ & $\begin{array}{r}\text { Peak stress } \\
\hat{\sigma}(\mathrm{MPa})\end{array}$ \\
\hline 0.14 & 3.0 & Aluminum & $77 \pm 9$ & $1.4 \pm 0.2$ & $140 \pm 20$ \\
0.14 & 1.3 & Aluminum & $15 \pm 1$ & $1.6 \pm 0.2$ & $115 \pm 15$ \\
0.25 & 3.0 & Aluminum & $79 \pm 6$ & $1.4 \pm 0.2$ & $100 \pm 15$ \\
0.25 & 2.0 & Aluminum & $30 \pm 4$ & $1.6 \pm 0.2$ & $95 \pm 15$ \\
0.25 & 1.3 & Aluminum & $16 \pm 1$ & $1.6 \pm 0.2$ & $85 \pm 15$ \\
1.0 & 3.0 & Aluminum & $74 \pm 9$ & $1.8 \pm 0.2$ & $85 \pm 15$ \\
0.25 & 1.4 & Steel & $38 \pm 3$ & $1.8 \pm 0.2$ & $105 \pm 15$ \\
0.25 & 0.9 & Steel & $18 \pm 2$ & $1.8 \pm 0.2$ & $90 \pm 15$ \\
0.25 & 0.7 & Steel & $12 \pm 1$ & $1.6 \pm 0.2$ & $85 \pm 15$ \\
\hline
\end{tabular}


Table 2.

Process parameters for the LMD1142 adhesive

\begin{tabular}{llllll}
\hline $\begin{array}{l}\text { Adhesive } \\
\text { layer } \\
\text { thickness }\end{array}$ & $\begin{array}{l}\text { Adherend } \\
\text { thickness } \\
h(\mathrm{~mm})\end{array}$ & Adherend & $\begin{array}{l}\text { Radius of } \\
\text { curvature } \\
(\mathrm{mm})\end{array}$ & $\begin{array}{l}\text { 'Intrinsic' } \\
\text { toughness } \\
\Gamma_{\mathrm{o}}\left(\mathrm{kJ} / \mathrm{m}^{2}\right)\end{array}$ & $\begin{array}{l}\text { Peak stress } \\
\hat{\sigma}(\mathrm{MPa})\end{array}$ \\
\hline 0.14 & 3.0 & Aluminum & $70 \pm 14$ & $1.8 \pm 0.2$ & $120 \pm 20$ \\
0.14 & 2.0 & Aluminum & $32 \pm 4$ & $2.0 \pm 0.2$ & $115 \pm 15$ \\
0.25 & 3.0 & Aluminum & $64 \pm 7$ & $2.3 \pm 0.2$ & $95 \pm 15$ \\
0.25 & 2.0 & Aluminum & $31 \pm 4$ & $2.0 \pm 0.2$ & $80 \pm 12$ \\
\hline
\end{tabular}

uniform silica spheres as spacers and ranged from 0.14 to $1.0 \mathrm{~mm}$. After curing and removing excess adhesive, the specimens were fractured by driving a thick wedge down the interface at $10 \mathrm{~mm} / \mathrm{min}$. The action of the wedge provided the bending moment required to fracture the specimens. During fracture, this bending moment also caused the adherends to bend plastically. The resultant radii of curvature were measured optically after the tests [7].

In situ optical observations of the adhesive layers were conducted during the tests by viewing the samples from the edge. Some typical micrographs are shown in Fig. 6. These observations indicated that the crack propagated continuously from its tip, with little or no damage nucleated ahead of it. When stress whitening (assumed to indicate crack-tip cavitation) was observed, it was limited to a region less than $100 \mu \mathrm{m}$ in size. These observations are consistent with Fig. 4a, which shows large stresses limited to a very small region ahead of the crack tip. Critical displacements across the interface at the crack tip were determined to be between about $10 \mu \mathrm{m}$ (for the thinnest layers of XD4600) and $40 \mu \mathrm{m}$ (for the thickest layers of LMD1142). Finally, it was noted that the fracture was always cohesive in these experiments, although the crack did occasionally run closer to one interface than the other. (Optical, post-fracture observations always showed a layer of adhesive on both fracture surfaces; however, frequently one surface retained more adhesive than the other.)

\subsection{EPZ calculations}

A numerical code incorporating an embedded-process zone (EPZ) along the interface has previously been developed to model this wedge test [4]. The analysis requires the use of a traction-separation law for the interface that is characterized by the two fracture parameters, $\Gamma_{\mathrm{o}}$ and $\hat{\sigma}$. As discussed in the Introduction, it is possible to validate the choice of the values of these two parameters by comparing numerical predictions for the deformed shapes of the failed specimens with the experimentally observed shapes. Details of the EPZ model and the numerical procedures used in this study are given in Yang et al. [4]. All geometries and combinations of materials that had been tested experimentally were modeled numerically. 
For each specimen, a series of EPZ calculations were done to determine the values of $\hat{\sigma}$ and $\Gamma_{\mathrm{o}}$ which allowed the predicted radius of curvature to match the experimentally observed radius.

The values of the fracture parameters that were obtained in this study are summarized in Tables 1 and 2. For the XD4600 adhesive, the 'intrinsic' toughness $\Gamma_{\mathrm{o}}$ was always in the range of $1.4-1.8 \mathrm{~kJ} / \mathrm{m}^{2}$. This was true for all thicknesses of the adherends and adhesive layers, and for both the steel and the aluminum samples. For the LMD1142 adhesive, $\Gamma_{\mathrm{o}}$ varied between 1.8 and $2.3 \mathrm{~kJ} / \mathrm{m}^{2}$. Beyond the fact that the 'intrinsic' toughnesses of the LMD1142 adhesive layers were somewhat higher than those of the XD4600 adhesive, there were no obvious trends associated with the values of toughness. For both adhesives, the peak stress appeared to vary between 80 and $140 \mathrm{MPa}$, with the lower limit corresponding to the thickest adhesive layers and thinnest adherends (i.e. when the constraint is lowest).

\section{DISCUSSION}

\subsection{Constrained deformation of the adhesive layer}

Stylized traction-separation laws are used in the EPZ model, but the essential elements are characterized by $\Gamma_{\mathrm{o}}$ and $\hat{\sigma}$. The continuum finite-element calculations discussed in Section 2.1 allow the maximum stress imposed by the adhesive layer on the adherends to be predicted as a function of $\mathcal{G}$, provided the deformation of the adhesive is adequately characterized. Since fracture is assumed to occur when the energy-release rate is equal to the 'intrinsic' toughness of the joint $\Gamma_{0}$, comparisons can be made between $\hat{\sigma}$ and the peak stress predicted by the continuum calculations at a value of $\mathcal{G}$ numerically equal to $\Gamma_{\mathrm{o}}$. Consistency between the two types of numerical calculations and the experimental observations would indicate that the adhesive layer can be modeled by using bulk constitutive properties in the constrained configuration up to the point of fracture. Failure to obtain selfconsistency between the different calculations and the experiments would indicate that the deformation mechanism of the adhesive layer had been fundamentally altered by the constraint. An alternative model for the deformation of the adhesive layer, invoking such effects as a large constraint effect on the yield stress, or the development of extensive damage, would then be required [9].

It was found that in all the cases studied here, the values of $\hat{\sigma}$ and $\Gamma_{\mathrm{o}}$ were consistent with the results of the continuum calculations. In other words, the peak stresses exerted by the adhesive layers when $\mathcal{G}=\Gamma_{\text {o }}$ were equal to the values of $\hat{\sigma}$ predicted by the EPZ model. This indicates that for these particular systems, the adhesive layers deformed by simple yielding in a constrained fashion as predicted from bulk data, with no other significant damage or deformation mechanisms being induced by the constraint. This is consistent with the in situ observations of the adhesive layer, which showed no evidence of macroscopic damage ahead of the crack tip. 


\subsection{Effect of the adherend material}

The properties of the adherend did not appear to affect the values of the 'intrinsic' toughness for these particular systems. The values of $\Gamma_{\mathrm{o}}$ were essentially the same for both the aluminum and the steel joints bonded with XD4600. This is a different conclusion from that made in an earlier paper [7] and requires further explanation. In ref. [7], an analytical expression derived from beam-bending theory was used to compute the toughness from the curvature. As shown by Yang et al. [4], if the wedge is too small, deformation of the arms is dominated by shear, rather than by bending. The resultant curvature is then smaller than it would have been had fracture occurred in response to a pure bending moment, and the use of the analytical expression to deduce the 'intrinsic' toughness results in an underestimate. However, another effect neglected by the bending analysis is that the cohesive stresses acting on the interface raise the moment required to cause fracture. This results in an overestimate of $\Gamma_{\mathrm{o}}$. These two limitations of the bending analysis act in opposite senses. Perhaps fortuitously, the results quoted in ref. [7] for aluminum appear to have been obtained under conditions where the two effects almost canceled each other out. However, it appears that the results for steel were obtained under conditions in which the shear effect was dominant, and the values of 'intrinsic' toughness that were obtained were too low. In the present work, not only was the size of the wedge chosen to ensure bending-dominated fracture, but the results were computed using an EPZ model so that both the effect of shear and the effect of the peak stress were rigorously included. The conclusion is that in these particular experiments, the 'intrinsic' toughness of the adhesive layer was not dependent on the material being bonded.

\subsection{Effect of constraint}

The results of Table 1 show that the 'intrinsic' toughness of the joint did not vary significantly when either the thickness of the adhesive layer or the thickness of the adherends was varied. In other words, the energy (per unit area of crack advance) dissipated in the adhesive layer was approximately independent of geometry. (This is in contrast to the total energy absorbed by the joint which includes the energy dissipated by plastic bending of the adherend, and which is sensitive to the thickness of the adherends.) While some variation in $\Gamma_{\mathrm{o}}$ was observed, it was at a level that was only just discernible, given the range of experimental uncertainty. In particular, there appeared to be no systematic dependence of the 'intrinsic' toughness on the adhesive layer thickness, despite a systematic decrease in peak stress with thicker adhesive layers. This is only possible if the critical displacements for failure also varied with layer thickness in such a way as to cancel the effects of a lower peak stress. A rough estimate for the energy absorbed by the adhesive layer (per unit area of crack advance) can be obtained from the product of the cohesive strength of the layer and the critical displacement. Hence, although an increase in adhesive layer thickness may result in less constraint and a lower peak stress, if it also results in a larger displacement before failure, then $\Gamma_{\mathrm{o}}$ may not vary with thickness. 
The interplay between cohesive stresses and critical displacements raises an interesting issue about the origin of $\Gamma_{\mathrm{o}}$. It was emphasized earlier that $\Gamma_{\mathrm{o}}$ and $\hat{\sigma}$ are the two fracture parameters governing the strength of a plastically-deforming joint; other characteristics of the cohesive tractions such as the shape of the curve and the critical displacement are of much less importance. $\Gamma_{\mathrm{o}}$ and $\hat{\sigma}$ are, therefore, the parameters used to characterize an adhesive layer when using the EPZ model. However, it should be noted that the 'intrinsic' toughness, which is the area under the traction-separation curve, is dictated by both the stress and the displacements. So, while the critical displacement is not an independent quantity in the EPZ model, it may be physically important in that it, along with $\hat{\sigma}$, dictates the value of $\Gamma_{0}$.

Unfortunately, there is at present no understanding of how to predict critical displacements from a priori calculations. However, they can be estimated from in situ measurements of the adhesive layer thickness at the crack tip during fracture (Fig. 6). These observations suggest values in the range of $10-20 \mu \mathrm{m}$ for the thinnest layers of XD4600 to 20-30 $\mu \mathrm{m}$ for the thicker layers, and from 20-30 $\mu \mathrm{m}$ for the thinnest layers of LMD1142 to 30-40 $\mu \mathrm{m}$ for the thicker layers. These values are consistent with the maximum displacements computed numerically, both in the continuum finite-element calculations and in the EPZ calculations. This supports the notion that the approximate constancy of the 'intrinsic' toughness arises from the compensating effects of peak cohesive stress and critical displacement. It should be emphasized that there may be other systems in which this compensation is not as balanced, in which case the toughness may exhibit a dependence on the adhesive layer thickness (either increasing or decreasing with thickness). Similar interactions between the peak stress and critical displacement may also play a role in the effect of strain rate on toughness. This is the subject of a separate study currently underway.

\subsection{A physical basis for traction-separation laws}

The agreement between the predicted and the observed displacements lends additional support to the assumption that the deformation of the adhesive layers can be modeled by continuum approaches in the particular cases considered here. This suggests that plots of the normal stress against displacement deduced from continuum finite-element calculations may be used to construct physically significant traction-separation laws for these joints. This is done by plotting values of the normal stress against the corresponding opening displacement at various positions ahead of the crack at an energy-release rate corresponding to $\Gamma_{\mathrm{o}} .{ }^{4}$ The results are shown in Figs $7 \mathrm{a}$ and $7 \mathrm{~b}$. In Fig. 7a, a direct comparison is made between the

\footnotetext{
${ }^{4}$ It is recognized that the continuum finite-element calculations presented here properly represent the expected conditions in the adhesive at the initiation of the crack. Some difference in the details might be expected in a steady-state configuration. However, it is not expected that such an effect will have a significant influence on the general results.
} 


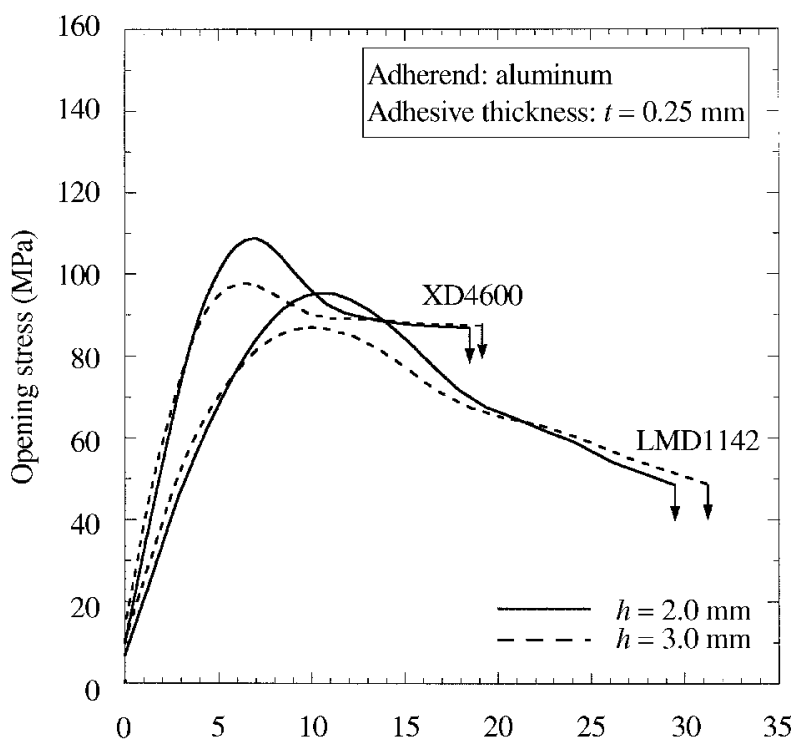

Opening displacement $(\mu \mathrm{m})$

(a)

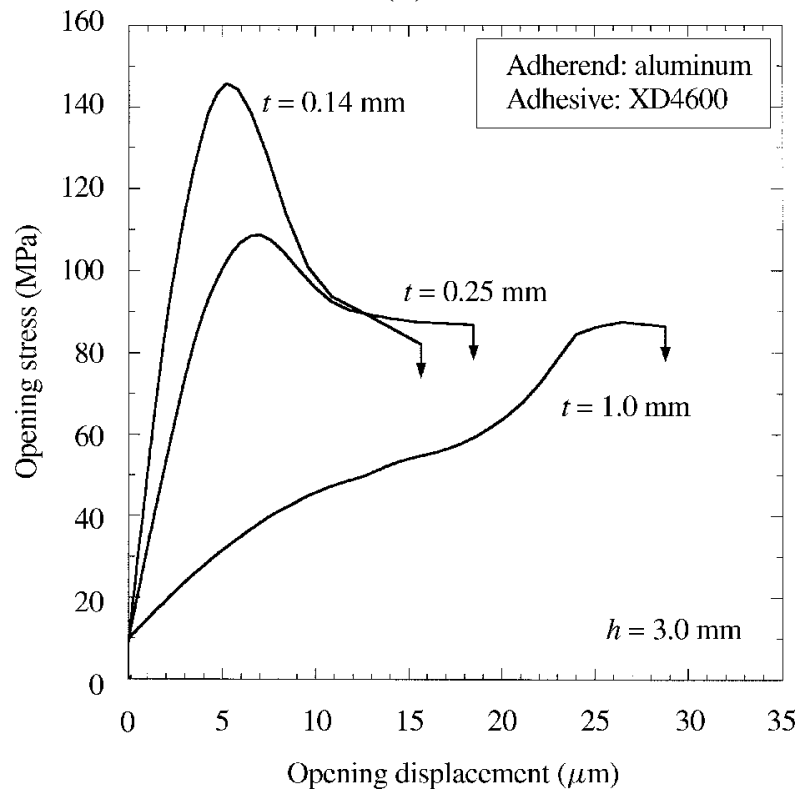

(b)

Figure 7. Examples of cohesive traction-separation laws inferred from the predicted relationship between the opening stresses and displacements for constrained adhesive layers. (a) Comparison between XD4600 and LMD1142 adhesive layers with identical geometries and, therefore, similar constraints. The thickness of the adhesive layer, $t$, in both cases is $0.25 \mathrm{~mm}$. Two curves are shown for each adhesive, one for an adherend thickness, $h$, of $2.0 \mathrm{~mm}$, and one for $h=3.0 \mathrm{~mm}$. (b) Illustration of the effect of the thickness of the adhesive layer, $t$, for the XD4600 adhesive bonded to $3 \mathrm{~mm}$ thick aluminum sheets. 
predicted traction-separation curves for the two adhesives subject to similar constraints. This figure illustrates that it is the difference in critical displacements that lies at the heart of why LMD1142 has a somewhat higher 'intrinsic' toughness than XD4600. Based on the constitutive properties, the tougher adhesive is expected to support a slightly lower stress, but the much higher critical displacements in this confined configuration (also observed experimentally) give rise to the higher toughness. In Fig. 7b, the effect of adhesive thickness is illustrated. As discussed in the previous section, the peak stress decreases for the thicker adhesive layers, but the critical displacement increases in such a fashion that $\Gamma_{\mathrm{o}}$ remains about constant.

\section{CONCLUSIONS}

The role of an adhesive layer can be regarded as providing cohesive tractions across an adhesive joint. Two key characteristics of these cohesive tractions are $\Gamma_{\mathrm{o}}$ and $\hat{\sigma}$, i.e. the area under the traction-separation curve and the peak stress, respectively. These two parameters dictate the strength and deformation of the joint. Their magnitude is dictated, in turn, by the deformation of the adhesive layer as it is constrained by the surrounding material. Furthermore, the two parameters can be used in an EPZ model to make quantitative predictions about the behavior of an adhesive joint.

The use of this approach has been demonstrated by investigating the deformation and fracture of adhesive joints made from steel and aluminum sheets bonded by two commercial adhesives. For these particular systems, the adhesive layers appeared to deform by simple yielding in a constrained fashion as predicted from bulk data, with no other significant damage or deformation mechanisms being induced by the constraint. The toughness of the joints was independent of the metal that they were made of, and there was no discernible trend in toughness with adhesive layer thickness. While the peak stress supported by the adhesive depended on the constraint, it appears that the maximum displacement also depended on the layer thickness in such a fashion that the total energy absorbed by the adhesive layer remained approximately constant.

\section{Acknowledgements}

This work was supported by NSF Grant CMS-9624452 and the Ford Motor Company.

\section{REFERENCES}

1. V. Tvergaard and J. W. Hutchinson, Philos. Mag. A70, 641-656 (1994).

2. V. Tvergaard and J. W. Hutchinson, J. Mech. Phys. Solids 44, 789-800 (1996).

3. V. Tvergaard and J. W. Hutchinson, J. Mech. Phys. Solids 40, 1377-1397 (1992).

4. Q. D. Yang, M. D. Thouless and S. M. Ward, J. Mech. Phys. Solids 47, 1337-1353 (1999). 
5. Q. D. Yang, M. D. Thouless and S. M. Ward, J. Adhesion 72, 115-132 (2000).

6. A. Needleman, J. Mech. Phys. Solids 38, 289-324 (1990).

7. M. D. Thouless, J. L. Adams, M. S. Kafkalidis, S. M. Ward, R. A. Dickie and G. L. Westerbeek, J. Mater. Sci. 33, 189-197 (1998).

8. B. C. Duncan, G. D. Dean and B. E. Read, Proceedings of Structural Adhesives in Engineering V Conference, pp. 274-279. Institute of Materials, Bristol (April 1998).

9. M. Cavalli, M. S. Kafkalidis, M. D. Thouless and S. M. Ward, manuscript in preparation. 